\title{
Image registration based on the structure tensor of the local phase
}

\author{
Zhang Li* ${ }^{\mathrm{a}}$, Lucas J. van Vliet ${ }^{\mathrm{a}}$, Jaap Stoker ${ }^{\mathrm{b}}$, Frans M. Vos ${ }^{\mathrm{a}, \mathrm{b}}$ \\ ${ }^{a}$ Quantitative Imaging Group, Department of Imaging Physics, Delft University of Technology,

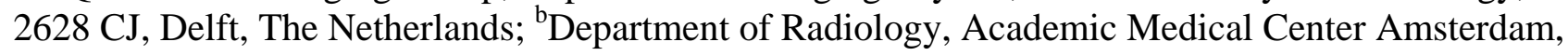 \\ 1105 AZ, Amsterdam, The Netherlands.
}

\begin{abstract}
Image registration of medical images in the presence of large intra-image signal fluctuations is a challenging task. Our paper addresses this problem by introducing a new concept based on the structure tensor of the local phase. The local phase is calculated from the monogenic signal representation of the images. The local phase image is hardly affected by unwanted signal fluctuations due to a space-variant background and a space-variant contrast. The boundary structure tensor combines the responses of edges and corners/junctions in one tensor, which has several advantages, compared to other structure tensors. We reorient the structure tensor during the registration by means of the finite-strain technique. The structure tensor is only calculated once during a preprocessing step. The results demonstrate that the proposed method effectively deals with large signal fluctuations. It performs significantly better than competing techniques.
\end{abstract}

Keywords: monogenic signal, tensor reorientation, structure-based registration

\section{INTRODUCTION}

Image registration is widely used for finding correspondence between medical images. The sum of squared differences (SSD), normalized cross covariance (NCC) and mutual information (MI) ${ }^{1,2}$ are standard (dis)similarity metrics used to measure correspondences. However, these metrics do not consider the local image structure, which may influence the robustness of non-rigid registration ${ }^{3}$. Several methods have integrated features of the local structure into the similarity metric $^{3,4,5}$ to improve the registration performance. Alternatively, the so-called MIND $^{6}$, DRAMMS ${ }^{7}$ and ROHE $^{8}$ techniques determine structural properties of image patches and apply a simple (dis)similarity metric (e.g. SSD).

However, none of the aforementioned methods is well designed for images with large intra-image signal fluctuations. These signal fluctuations are often observed in medical images (e.g. the bias field in magnetic resonance imaging) and introduce "uncharacteristic" information. Accidentally treating this "uncharacteristic" information as local structure may give rise to incorrect registration.

We encountered this problem upon registering three-dimensional Dynamic Contrast Enhanced MR images- to postcontrast abdominal MRI data for quantifying Crohn's disease activity. This is relevant since the two modalities contain complementary information and both suffer from large signal fluctuations ${ }^{9}$. We present a new, efficient structure representation based on the Structure Tensor of the lOcal Phase (STOP) in order to overcome the problem with the signal fluctuations. The local phase is derived from the monogenic signal representation of the image ${ }^{10}$, which is insensitive to signal fluctuations. Furthermore, the structure tensor is a well-known concept representing the image structure. The registration results will demonstrate that this new approach outperforms other structure-based methods.

Medical Imaging 2015: Image Processing, edited by Sébastien Ourselin, Martin A. Styner, Proc. of SPIE Vol. 9413, $941330 \cdot$ (C) 2015 SPIE · CCC code: 1605-7422/15/\$18 $\cdot$ doi: 10.1117/12.2081840 


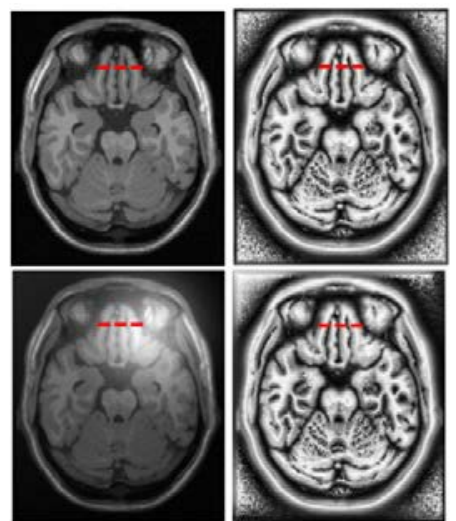

(a)

(b)

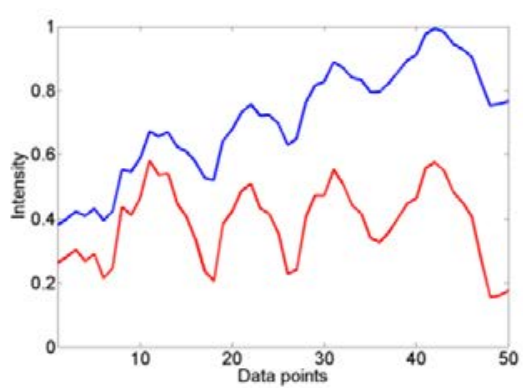

(c)

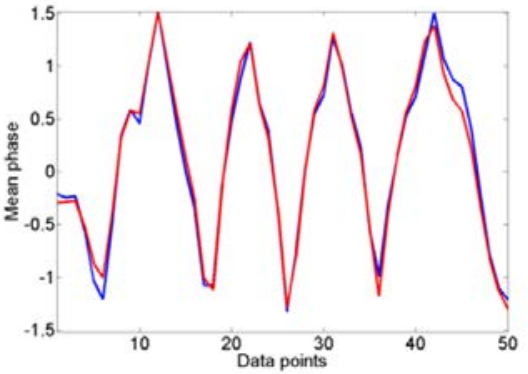

(d)

Figure 1. (a) Top: a brain MR image; bottom: same image deformed by a simulated local intensity distortion; (b) mean phase images from (a); (c) intensity profiles along the red dashed lines in (a): the red line is from the original image, the blue line from the deformed image; (d) similar intensity profiles from the corresponding mean phase images.

\section{METHODOLOGY}

\subsection{Monogenic signal and local phase}

The mean phase of a multi-dimensional image is derived from the monogenic signal ${ }^{10}$. Essentially, the monogenic signal extends the analytic signal from 1-D to $N$-D by means of the Riesz transform. The monogenic signal for 2-D images (expressed in quaternions) is defined as:

$$
f_{M}(\mathbf{x})=f_{e}(\mathbf{x})-(i, j) \mathbf{f}_{R}(\mathbf{x}),
$$

where $f_{e}(\mathbf{x})$ is the even component of the original image calculated by convolving the original image with a rotationally symmetric, real-valued, zero-mean band-pass filter; the vector-image $\mathbf{f}_{R}(\mathbf{x})$ holds the odd components of the aforementioned band-pass filtered image. The local phase image is then calculated over multiple $(n)$ scales of the filter ${ }^{11}$ :

$$
\bar{\varphi}(\mathbf{x})=\operatorname{atan}\left(\frac{\sum_{i=1}^{n}\left|\mathbf{f}_{R}^{i}(\mathbf{x})\right|}{\sum_{i=1}^{n} f_{e}^{i}(\mathbf{x})}\right),
$$

in which $i$ denotes the scale. In our work, we use a log-Gabor filter, because it is a zero-DC filter with a tunable bandwidth. An example of a local phase image calculated from an image (Figure 1(a)) is shown in Figure 1(b). Observe that the local phase is invariant to intensity distortions.

\subsection{Structure tensor and STOP representation}

The structure tensor is often used for characterizing the local image structure. Several types of structure tensors were proposed for different applications. We choose the boundary tensor ${ }^{12}$ for our registration framework. The boundary tensor is defined as

$$
T=T_{o}+\frac{1}{4} T_{e},
$$

where $T_{o}$ and $T_{e}$ are tensors representing the odd and even image structures respectively. Effectively, this method integrates edge and corner/junction detection into a single tensor representation. Since the odd and even components of the tensor are by definition in quadrature, a boundary tensor implicitly allows us to incorporate all relevant structural information - edges (odd) and lines (even) - into a single tensor.

The so-called STOP representation for images is obtained by first deriving the local-phase image via (2), after which the structure tensor (3) of the local-phase image is computed.

\subsection{Structure tensor reorientation}

Tensor reorientation has already been incorporated into the registration of diffusion tensor images ${ }^{13}$. It is used to adjust the tensor orientation during registration. Similar to diffusion tensor reorientation, we can also adapt the structure tensor 
during registration. A standard way to do so is the finite strain (FS) strategy. FS computes a rigid rotation matrix from the deformation gradient as follows:

$$
R(\mathbf{x})=\left(J(\mathbf{x}) J(\mathbf{x})^{T}\right)^{-\frac{1}{2}} J(\mathbf{x}),
$$

where $R(\mathbf{x})$ is the rotation matrix in a pixel and $J(\mathbf{x})$ the Jacobian matrix associated with the geometric transformation at pixel $\mathbf{x}$. Then, the structure tensor $T(\mathbf{x})$ is reoriented by:

$$
T^{\prime}(\mathbf{x})=R^{T}(\mathbf{x}) T(\mathbf{x}) R(\mathbf{x}),
$$

where $T^{\prime}(\mathbf{x})$ is the reoriented structure tensor. Incorporating this reorientation strategy into the registration framework allows us not only to improve the registration by incorporating structural information, it may also reduce the computational complexity because the structure tensor only needs to be calculated once during the preprocessing stage.

\subsection{Registration framework}

Since the STOP representation is invariant to local signal fluctuation, we can use the Frobenius norm to calculate a dissimilarity metric based on this tensor representation:

$$
\sum_{\mathbf{x}} \sqrt{\operatorname{trace}\left(\left(T_{f}(\mathbf{x})-T_{m}\left(\mathbf{x}+\mathbf{u}_{m}\right)\right)\left(T_{f}(\mathbf{x})-T_{m}\left(\mathbf{x}+\mathbf{u}_{m}\right)\right)^{\mathrm{T}}\right)},
$$

where $T_{f}$ and $T_{m}$ are tensor representations of respectively the fixed and moving images and $\mathbf{u}_{m}$ is the deformation field for each pixel. Gauss-Newton optimization is used for minimizing (6) with the total variation of the deformation field $\mathbf{u}_{m}$ as regularization term. The tensor reorientation is embedded in the registration framework.

\section{RESULTS}

We compared our STOP methods with two state-of-the art structure-based registration methods: DRAMMS and MIND. Two datasets were used for the comparison: (1) synthetically distorted MRI brain data and (2) abdominal MR images.

\subsection{Synthetic brain data}

We simulated images by applying an intensity distortion to brain MRI data as described by Myronenko et al. ${ }^{14}$. First, a spatially varying intensity distortion was applied to a brain MR image to create the fixed image (see Figure 2, first column). Second, another intensity distortion was applied to the same original brain image in a comparable manner. Thereafter, a geometric distortion with a thin-plate spline (TPS) model was applied to this image to get a second image, the moving image (see Figure 2, second column). The registrations of the moving image to the fixed image by the three different methods are shown in the remaining images of Figure 2. The DRAMMS and MIND methods were applied using their default settings. One can conclude from Figure 2 that only the proposed method has the ability to deal with images suffering from signal fluctuation (see the regions pointed out by yellow arrows).

We randomly generated 100 different moving images to further evaluate the methods. Subsequently, we calculated the root mean square difference (RMSD) between the imposed and estimated spatial deformations by the different registration methods. The results are shown in Table I. One can see that the STOP method gave the smallest RMSD, which was significantly better than the other methods as assessed by a two-sided signed rank test $(\mathrm{p}<0.05)$.

\subsection{Abdominal MR images}

Abdominal MR datasets of 30 patients from a previous study into Crohn's disease ${ }^{15}$ were used to evaluate our registration method. Our aim was to register a DCE-MRI series to a post-contrast MR image. Time intensity curves (TICs) from the DCE-MRI series contain important information about the disease activity ${ }^{16}$. Diseased regions were delineated on the post-contrast MR image, which had higher resolution (see Figure 2, second and third rows). In the previous study these annotations were used to measure the thickness of the bowel wall and the length of the diseased part. Accurate registration between DCE- and post-contrast MRI allows combining complementary information within the annotated regions. The DCE-MRI series (450 volumes) were firstly registered to themselves by using the method described $i^{16}$. This left around 100 registered volumes and one expiration phase DCE-MRI template for each patient. 

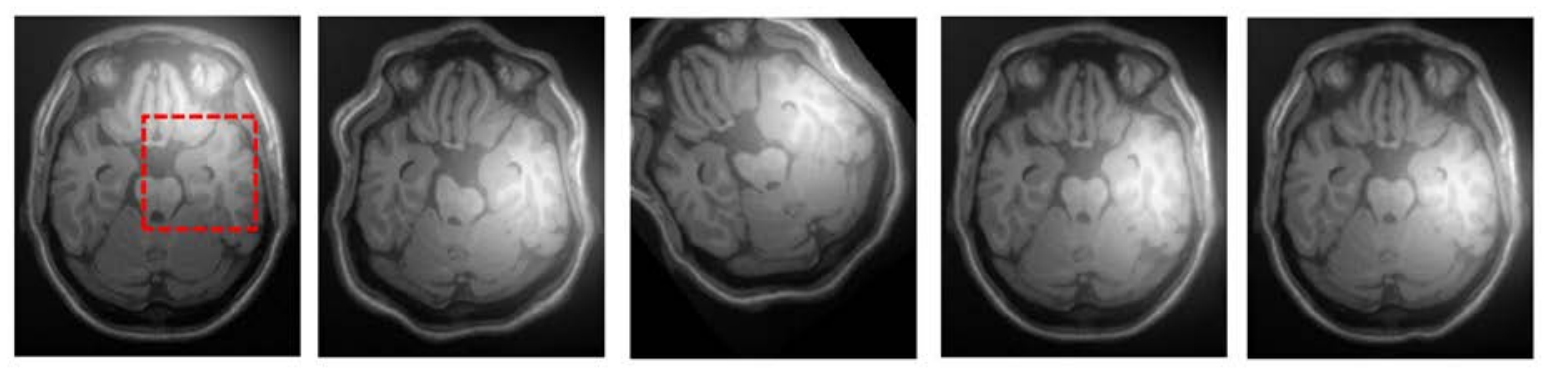

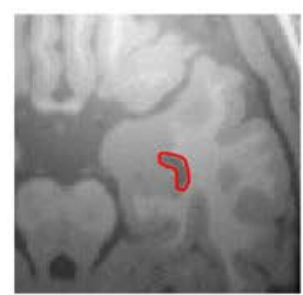

fixed image

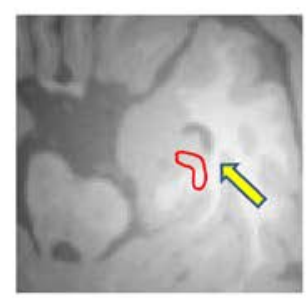

moving image

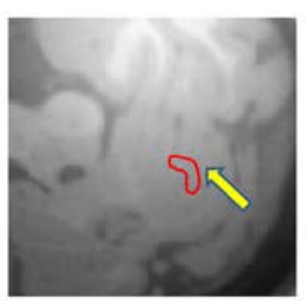

DRAMMS

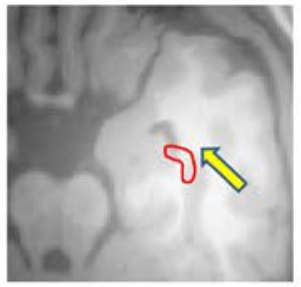

MIND

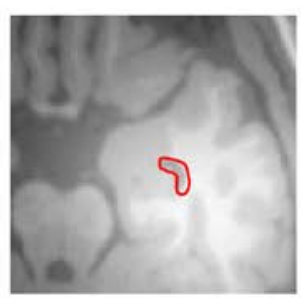

STOP

Figure 2. Results of registering synthetically deformed brain images using different methods; the first row contains: fixed image, moving image, and the registration outcomes using the DRAMMS, MIND and STOP methods; the second row shows a detail with signal fluctuations. A region of interest is annotated (red contour) on the fixed image. This region is copied to the moving image and the registration outcomes by the three methods. he yellow arrows indicate the registration errors. Note that the outcome of the STOP method is highly acceptable.

Table I. Evaluation of the registration performance on synthetic brain data by three methods: DRAMMS, MIND and STOP. The performance was measured by the root mean square difference (RMSD) between imposed deformations and estimated deformations over 100 cases. The numbers report the mean value and the standard deviation (std) between brackets. The unit is mm and the number printed in boldface indicates the best result.

\begin{tabular}{|l|l|l|l|l|}
\hline & no registration & DRAMMS & MIND & STOP \\
\hline RMSD & $4.28(0.43)$ & $7.56(1.77)$ & $1.46(0.30)$ & $\mathbf{1 . 3 2}(\mathbf{0 . 1 9 )}$ \\
\hline
\end{tabular}

The DCE-MRI template image was registered to the post-contrast MR image to obtain spatial alignment. The three registration methods as before were used to do so. A visual comparison is shown in Figure 3. An experienced radiologist annotated two diseased regions on the post-contrast MR image (red polygons). The annotations were then copied to the DCE-MRI template prior to and after registration by DRAMMS, MIND and STOP. The yellow arrows point out the registration errors. One can see that DRAMMS failed in regions with strong signal fluctuations (e.g. second row: the bladder; third row: the bowel wall). MIND already facilitated improved registration in those regions. However, errors still occurred around the bowel wall, because MIND is not fully invariant to signal fluctuations, which makes that this method gets trapped in a local minimum.

After registering the DCE-MRI template to the post-contrast MR image, we applied the found deformation field to the entire set of pre-aligned DCE-MR images. Subsequently, TICs were derived from the annotated diseased regions copied from the post-contrast MR image. A tri-exponential function was fit to the TIC after of which one fit parameter called $A_{1}$ reflects the strong upward slope of the TIC ${ }^{16}$. We correlated this feature to the Crohn's Disease Endoscopic Index of Severity, CDEIS ${ }^{17}$. The results are shown in Table II. It turns out that the STOP method gave the highest and one-andonly significant correlation $(\mathrm{r}=0.53, \mathrm{p}=0.03)$. 

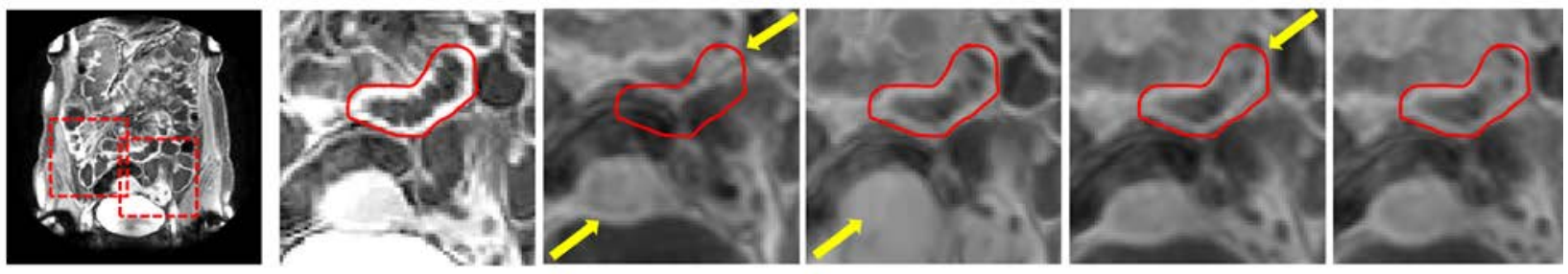

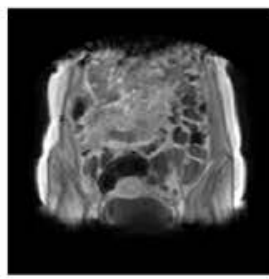

(a)

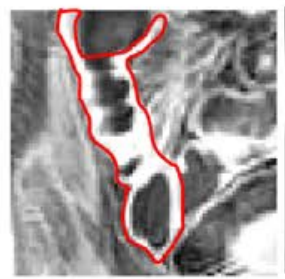

(b)

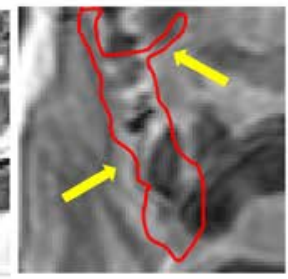

(c)

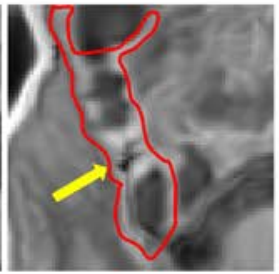

(d)

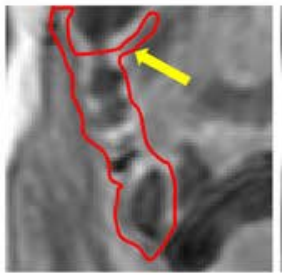

(e)

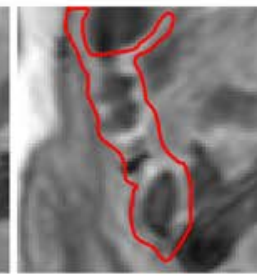

(f)

Figure 3. Results of registering abdominal MR images. (a) Fixed image (post-contrast, top) and moving image (DCE-MR image, bottom); (b) two pieces of diseased bowel in two regions of interests (ROIs) were annotated with a red contour on the post-contrast images; the ROIs correspond to the red-dashed boxes in (a). (c)-(f) show the annotations from (b) copied to the DCE-MR image prior to and after registration by means of the DRAMMS, MIND and STOP methods. The yellow arrows indicate registration errors.

Table II. Correlation coefficients (CC) of an extracted DCE-MRI image feature for contrast enhancement and CDEIS (ground truth) prior to registration, and after registration by DRAMMS, MIND and STOP. The p-values of the correlations are shown between brackets. The number printed in boldface indicates the best result.

\begin{tabular}{|c|c|c|c|c|}
\hline & no registration & DRAMMS & MIND & STOP \\
\hline CC & $0.39(0.12)$ & $0.41(0.10)$ & $0.43(0.08)$ & $\mathbf{0 . 5 3}(\mathbf{0 . 0 3})$ \\
\hline
\end{tabular}

\section{CONCLUSION}

We proposed a new local structure representation based on the structure tensor of the local phase image. It facilitates image registration in the presence of large intra-image signal fluctuations. The registration results that we obtained demonstrated that the new technique outperformed state-of-art structure-based methods. The proposed framework can be adapted to different registration purposes by using a different type of structure tensor (e.g. gradient tensor) or different measures of the local phase (e.g. phase congruency).

\section{REFERENCES}

[1] Maes, F., Collignon, A., Vandermeulen, D., Marchal, G., and Suetens, P. , "Multimodality image registration by maximization of mutual information," IEEE Trans. Med. Imag.16(2), 187-198(1997).

[2] Viola, P., and Wells W.M.I.I.I. , "Alignment by maximization of mutual information," Int. J. Comput. Vision 24(2), 137-154(1997).

[3] Yi, Z., and Soatto, S, "Multimodal Registration via Spatial-Context Mutual Information," Proc. Inf. Process Med. Imaging (IPMI), 424-435(2011). 
[4] Gorbunova, V., Lol, P., Ashraf, H., Dirksen, A., Nielsen, M., and de Bruijne, M., "Weight Preserving Image Registration for Monitoring Disease Progression in Lung CT," Proc. Med. Image Computing Computer Assist. Intervent. (MICCAI), 863-870(2008).

[5] Song, G., Tustison, N., Avants, B., and Gee, J., "Lung CT Image Registration Using Diffeomorphic Transformation Models," Proc. Medical Image Analysis for the Clinic: A Grand Challenge, 23-32(2010).

[6] Heinrich, M. P., Jenkinson, M., Bhushan, M., Matin, T., Gleeson, F. V., Brady, S. M., and Schnabel, J. A. , "MIND: modality independent neighbourhood descriptor for multi-modal deformable registration," Med. Image Anal.16(7), 1423-1435(2012).

[7] Ou, Y., Sotiras, A., Paragios, N., and Davatzikos, C., "DRAMMS: Deformable registration via attribute matching and mutual-saliency weighting," Med. Image Anal.15(4), 622-639(2011).

[8] Li, Z., van Vliet, L. J., and Vos, F. M., "Self Similarity Image Registration Based on Reorientation of the Hessian," Proc. Abdominal Imaging. Computation and Clinical Applications, 20-28(2013).

[9] Li, Z., van Vliet, L. J., and Vos, F. M., "Image registration based on autocorrelation of local structure," submitted(2015).

[10] Felsberg, M., and Sommer, G., "The monogenic signal," IEEE Trans. Signal Process.49(12), 3136-3144(2001).

[11] Kovesi, P., "Phase congruency detects corners and edges," Proc. DICTA, 309-318(2003).

[12] Kothe, U., "Integrated edge and junction detection with the boundary tensor," Proc. IEEE International Conference on Computer Vision (ICCV), 424-431(2003).

[13] Yeo, B., Vercauteren, T., Fillard, P., Peyrat, J., Pennec, X., Golland, P., Ayache, N., and Clatz, O.,"Dt-refind: Diffusion Tensor Registration with Exact Finite Strain Differential," IEEE Trans. Med. Imag.28(12), 1914-1928(2009).

[14] Myronenko, A., and Song, X., "Image Registration by Minimization of Residual Complexity," Proc. Computer Vision and Pattern Recognition (CVPR), 49-56(2009).

[15] Vos, F. M., Tielbeek, J. A., Naziroglu, R. E., Li, Z., Schueffler, P., Mahapatra, D., Wiebel, A., Lavini, C., Buhmann, J. M., Hege, H.-C., Stoker, J., and van Vliet, L. J., "Computational Modeling for Assessment of IBD: to be or not to be?," Proc. 34th Annual International Conference of the IEEE EMBS, 3974-3977(2012).

[16] Li, Z., Tielbeek, J. A. W., Caan, M. W. A., Puylaert, C. A.J., Ziech, M. L. W., Nio, C., Stoker, J., van Vliet, L. J., and Vos, F. M. , "Expiration-Phase Template-based Motion Correction of Free-Breathing Abdominal Dynamic Contrast Enhanced MRI," IEEE Trans. Biomed. Eng., in press(2015).

[17] Mary, J. Y., and Modigliani, R., "Development and validation of an endoscopic index of the severity for Crohn's disease: a prospective multicentre study. Groupe d'Etudes Thérapeutiques des Affections Inflammatoires du Tube Digestif (GETAID)," Gut 30(7), 983-989(1989). 